\title{
Gestão de recursos humanos e desempenho operacional: evidências empíricas
}

\author{
Human resource management and operational \\ performance: empirical evidence
}

\author{
Charbel José Chiappetta Jabbour ${ }^{1}$ \\ Wesley Ricardo de Souza Freitas ${ }^{1}$ \\ Adriano Alves Teixeira ${ }^{1}$ \\ Ana Beatriz Lopes de Sousa Jabbour ${ }^{1}$
}

\begin{abstract}
Resumo: A gestão de recursos humanos é frequentemente apontada como uma variável que influencia positivamente no desempenho das organizações. Enquanto argumentos e proposições teóricas de teor similar se multiplicam na literatura especializada, ainda são raras as pesquisas que lançam luzes sobre essa relação para empresas brasileiras. Nesse sentido, foi realizada uma pesquisa com o objetivo de verificar, empiricamente, se a gestão de recursos humanos influencia de maneira positiva o desempenho de empresas do segmento de autopeças e componentes automotivos em suas operações. Metodologicamente, realizou-se um survey com 75 empresas do setor mencionado, cujos dados foram analisados por meio de modelagem de equações estruturais, uma análise multivariada de segunda geração. A principal hipótese da pesquisa foi confirmada, revelando que, de fato, a gestão de recursos humanos está positivamente relacionada com o desempenho operacional das empresas analisadas.
\end{abstract}

Palavras-chave: Gestão de recursos humanos. Desempenho operacional. Setor de autopeças e componentes automotivos. Modelagem de equações estruturais. Brasil.

\begin{abstract}
Human resource management is often cited as a variable that positively influences the performance of organizations. Arguments and theoretical proposals of similar content have increased in the literature, yet there is very little research that sheds light on this relationship in Brazilian companies. Accordingly, a survey was conducted in order to check empirically whether the human resource management positively influences the operational performance of companies in the Brazilian automotive industry focusing on the segment of automotive parts and components. Methodologically, a survey of 75 companies in the sector aforementioned was carried out; the data were analyzed using Structural Equation Modeling, a second-generation multivariate analysis. The main hypothesis was confirmed indicating that human resource management is indeed positively related to the operational performance of the companies studied.
\end{abstract}

Keywords: Human resource management. Operational performance. Automotive parts and automotive component sector. Structural equation modeling. Brazil.

\section{Introdução}

As organizações empresariais possuem na essência de sua definição um coletivo de indivíduos unidos pela busca de um determinado desempenho (HALL, 2004). Portanto, tendem a ser fundamentalmente influenciadas pelas pessoas que nelas trabalham. Nesse sentido, Vergara (2011) afirma que as organizações, quando analisadas sem as pessoas que delas tomam parte, nada mais são do que instalações e prédios abandonados. Por isso gerenciar esse coletivo de indivíduos é relevante para as organizações.

Entretanto, o nome dado a esse coletivo de indivíduos que formam as organizações vem mudando com o avanço da história. De "empregados" a

"funcionários", de "público interno" a "colaboradores", verifica-se que o foco da área de gestão de recursos humanos é induzir o desempenho humano ao nível de excelência para a consecução dos objetivos organizacionais. Como afirmam Boudreau et al. (2003), a gestão de recursos humanos é tão importante para o bom desempenho das organizações manufatureiras quanto às melhorias e atualizações tecnológicas por elas adotadas.

Como consequência desse objetivo inerente à gestão de recursos humanos - de contribuir com o desempenho organizacional - diversos pesquisadores afirmam que existe um relacionamento positivo

\footnotetext{
${ }^{1}$ Departamento de Engenharia de Produção, Faculdade de Engenharia, Universidade Estadual Paulista - UNESP, Av. Engenheiro Luiz Edmundo Carrijo Coube, 14-01, CEP 17033-360, Vargem Limpa, Bauru, SP, Brasil. e-mail: prof.charbel@ gmail.com
} 
entre as práticas da gestão de recursos humanos e o desempenho das organizações (SCHULER; JACKSON, 1987; DYER; REEVES, 1995; HUSELID, 1995; BECKER; GERHART, 1996; ULRICH, 1997; BECKER; HUSELID, 2006; JANSSENS; STEYAER, 2009; PAAUWE, 2009; FERGUSON; REIO JUNIOR., 2010). Esse argumento é também evidenciado, de forma teórica, em algumas das principais publicações sistematizadas sobre recursos humanos no Brasil (FLEURY, 2002; DUTRA, 2004; MARRAS, 2001; LIMONGI-FRANÇA, 2007; DUTRA, 2008; SILVEIRA et al. 2010). Entretanto, ainda são raras as pesquisas nacionais que oferecem evidências empíricas suficientes para corroborar a proposição teórica de que a gestão de recursos humanos contribui positivamente para o desempenho organizacional.

Dessa lacuna, surge uma necessidade de compreender, de maneira empírica e com base em dados práticos, se realmente a gestão de recursos humanos, por meio de suas principais práticas (recrutamento e seleção, treinamento, avaliação de desempenho, recompensas e benefícios), está correlacionada com as medidas de desempenho operacional das organizações (como, por exemplo, custo, qualidade, flexibilidade, lançamento de produtos etc.). Acredita-se que, se a relação entre recursos humanos e desempenho é verídica, ela tende a se manifestar como tal no setor de autopeças e componentes automotivos, uma vez que esse setor industrial procura antecipar boas práticas de gestão, afetando toda a cadeia produtiva (WOMACK; JONES; ROOS, 2004).

Como consequência, a dúvida que motiva esta pesquisa pode ser assim descrita: estaria a gestão de recursos humanos influenciando positivamente o desempenho operacional de empresas do segmento de autopeças e componentes automotivos? Objetiva-se, portanto, verificar empiricamente se a gestão de recursos humanos influencia positivamente $o$ desempenho das operações de empresas do segmento de autopeças e componentes automotivos. De forma mais específica, almeja-se, com esta pesquisa:

- Propor um framework relacional entre gestão de recursos humanos e desempenho operacional em empresas brasileiras do segmento de autopeças e componentes automotivos;

- Realizar um survey com empresas do setor de autopeças e componentes automotivos;

- Verificar quais são as principais práticas que compõem a gestão de recursos humanos desse setor;

- Verificar quais são as principais medidas de desempenho operacional desse setor;

- Testar a hipótese de relacionamento positivo entre recursos humanos e desempenho no setor escolhido, por meio de modelagem de equações estruturais.
Este artigo contempla seis seções, sendo a primeira Introdução, a qual contextualiza a questão de pesquisa e delimita os objetivos do artigo. A segunda, Fundamentação teórica, apresenta definições e estudos anteriores para destacar of framework e as hipóteses da pesquisa. A terceira, Procedimentos metodológicos, apresenta a forma de coleta e análise de dados e justifica a escolha do objeto de estudo. A quarta são os Resultados da pesquisa. A quinta e a sexta seção são, respectivamente, a Discussão dos resultados e as Conclusões da pesquisa.

\section{Fundamentos conceituais, framework e hipóteses da pesquisa}

\subsection{Gestão de recursos humanos (GRH)}

A moderna gestão de recursos humanos (GRH) teve origem, como objeto de estudo e ensino, entre o final da década de 1970 e o início da de 1980, nos Estados Unidos (BREWSTER; MORLEY; BUCIUNIENE, 2010). Apesar da gestão de recursos humanos ter sempre existido, somente a partir do surgimento das ciências comportamentais reconheceu-se o real valor da GRH, principalmente devido ao reflexo de suas práticas no desempenho das organizações (SCROGGINS; BENSON, 2010).

A GRH é entendida como um conjunto de atividades para a adequada gestão dos funcionários (BOSELIE; DIETZ; BOON, 2005). Fischer (2002) refere-se à gestão de pessoas como a maneira pela qual uma empresa organiza, gerencia e orienta o comportamento das pessoas. Nesse sentido, Milkovich e Boudreau (2006, p. 19) a conceituam como "uma série de decisões integradas que formam as relações de trabalho e influenciam diretamente a capacidade da organização e de seus agentes de atingir seus objetivos".

Naturalmente, todas as organizações têm que gerir pessoas, no entanto, não podem fazê-lo da mesma forma em todos os contextos (BREWSTER; MORLEY; BUCIUNIENE, 2010; GUEST, 2011). As variações contextuais podem promover melhorias ou restrições sobre as práticas de GRH de determinada organização ou de setores industriais específicos (JACKSON; SCHULER, 1995).

Para fazer frente a alguns argumentos de que a área de $\mathrm{RH}$ pouco contribui com as organizações, houve, em meados de 1990, uma profusão de afirmações universais sobre a correlação positiva entre GRH e desempenho das organizações (LENGNICK-HALL et al., 2009). Entretanto, pesquisas recentes (DEWETTINCK; REMUE, 2011) revelam que essa relação tende a ser influenciada por fatores culturais, econômicos, políticos e sociais dos países em que as organizações atuam. Assim, a simples existência de práticas de GRH não garante sua contribuição para a eficácia 
organizacional, sendo essa contingencial (BOSELIE; DIETZ; BOON, 2005).

O principal desafio da GRH é, portanto, alinhar as suas principais práticas, definidas no Quadro 1, à estratégia e desempenho das organizações (LENGNICK-HALL et al., 2009).

\subsection{A influência da GRH no desempenho organizacional}

Os recursos humanos das organizações - bem como as práticas para sua adequada gestão - são elementos essenciais para uma vantagem competitiva sustentada (JAMROG; OVERHOLT, 2004; VOORDE; PAAUWE; VELDHOVEN, 2010). Nesse sentido,

[...] parece haver evidências suficientes para afirmar que algumas práticas de recursos humanos, isoladamente ou em conjunto, podem trazer melhores resultados para a organização. (LACOMBE; ALBUQUERQUE, 2008, p. 7).

Essa afirmação é confirmada por diversas outras pesquisas que destacaram a relação entre a GRH e o desempenho organizacional: Arthur (1994), Huselid (1995), Becker e Gerhart (1996), Delaney e Huselid (1996), Deleey e Doty (1996), Wright, Gardner e Moynihan (2003), por exemplo. O pressuposto fundamental dessas pesquisas é que o desempenho organizacional pode ser influenciado positivamente pelo conjunto de práticas de GRH (HUSELID; JACKSON; SCHULER, 1997).
Na vertente mais moderna e estratégica, a GRH é conceituada como sendo o projeto e a combinação de práticas gerenciais de pessoas orientadas para a melhoria da eficácia organizacional (BOSELIE; DIETZ; BOON, 2005) na busca de ligações entre as políticas de GRH e o desempenho organizacional (KOUHY; VEDD; YOSHIKAWA, 2009). Nesse sentido, Cabrera e Cabrera (2003) destacam que, assim como a gestão de marketing precisa saber o efeito de uma campanha de vendas no volume de vendas, a área de GRH precisa saber o efeito das suas práticas sobre os resultados organizacionais. Esse é o grande desafio para a gestão de recursos humanos: avaliar e orientar o alinhamento da gestão de recursos humanos com a gestão organizacional a fim de obter eficácia nos resultados (JAMROG; OVERHOLT, 2004).

Boselie, Dietz e Boon (2005) investigaram, entre 1994 e 2003, 104 artigos publicados em journals para conhecer a "caixa-preta da GRH", ou seja, as relações entre a GRH e o desempenho organizacional. Observaram que não existe um quadro consistente de resultados para tal fenômeno. Apesar das evidências de que a GRH influencia o desempenho organizacional, pouco se sabe sobre quais são as práticas de recursos humanos que são ou não vinculadas a um desempenho organizacional superior. Portanto, ainda não está claro como os recursos humanos dentro de uma organização agregam valor ao desempenho (VOORDE; PAAUWE; VELDHOVEN, 2010).

Quadro 1. Práticas de gestão de recursos humanos.

\begin{tabular}{|c|c|}
\hline Práticas & Conceitos \\
\hline $\begin{array}{l}\text { Recrutamento e } \\
\text { seleção }\end{array}$ & $\begin{array}{l}\text { "Recrutamento consiste no processo de atração de novas pessoas para os quadros da empresa e } \\
\text { a seleção consiste na escolha das pessoas certas." (DESSLER, 2003, p. 106) }\end{array}$ \\
\hline Treinamento & $\begin{array}{l}\text { É uma ação organizacional planejada, que possibilita a aquisição de habilidades técnicas e } \\
\text { comportamentais, assim como contribui para o desenvolvimento de estratégias cognitivas que } \\
\text { podem tornar o indivíduo mais apto a desempenhar suas funções, atuais ou futuras (BORGES- } \\
\text { ANDRADE, 2002). }\end{array}$ \\
\hline $\begin{array}{l}\text { Avaliação de } \\
\text { desempenho }\end{array}$ & $\begin{array}{l}\text { As avaliações podem ser definidas como um processo que visa determinar os resultados do } \\
\text { trabalho de um funcionário e uma de suas principais funções é oferecer uma justificativa para } \\
\text { a compensação dos seus resultados e esforços (TÜRK; ROOLAHT, 2007). A avaliação de } \\
\text { desempenho é um processo formalizado para classificar o desempenho atual do funcionário } \\
\text { (STONER; FREEMAN, 1999), além de ser "[...] utilizada para tomar decisões fundamentais } \\
\text { relativas às pessoas, tais como promoções, transferências e rescisões; para identificar as } \\
\text { necessidades de treinamento; para dar feedback aos funcionários sobre como a organização } \\
\text { encara seu desempenho e, frequentemente, é utilizada como base para os reajustes salariais." } \\
\text { (ROBBINS; DECENZO, 2004, p. 246). }\end{array}$ \\
\hline Recompensas & $\begin{array}{l}\text { O termo refere-se a "[...] todos os pagamentos monetários e todos os bens ou mercadorias } \\
\text { usados para recompensar empregados." (DAFT, 1999, p. 263). As decisões sobre a estruturação } \\
\text { e o funcionamento do subsistema de recompensas e seu gerenciamento no dia a dia } \\
\text { organizacional constituem uma das dimensões mais críticas em gestão de pessoas (HIPOLITO, } \\
\text { 2002). }\end{array}$ \\
\hline Benefícios & $\begin{array}{l}\text { "São facilidades e conveniências compartilhadas pela organização e pelos funcionários que não } \\
\text { estão contempladas no salário direto." (OLIVEIRA; LEONE, 2008, p. 108). "Estão divididos } \\
\text { entre aqueles exigidos por lei e os que são opcionais para o empregador." (BATEMAN; } \\
\text { SNELL, 1998, p. 293). }\end{array}$ \\
\hline
\end{tabular}


Em outro esforço acadêmico para mensurar o impacto da GRH, Gooderham, Ringdal e Parry (2006), realizaram um grande levantamento com 3.281 empresas de 16 países da Europa, a fim de verificar a influência das práticas de $\mathrm{RH}$ "calculistas" (que visam melhorar a eficiência a partir de critérios mensuráveis e do controle das pessoas) e "colaborativas" (orientada por laços psicológicos entre os objetivos organizacionais e individuais) sobre o desempenho de empresas. Os autores concluíram que o pacote de práticas "calculistas" tem maior impacto sobre o desempenho da empresa do que as práticas "colaborativas". Assim, as várias maneiras com que as organizações configuram seu conjunto de práticas de recursos humanos têm importantes consequências nas atitudes e comportamentos dos trabalhadores e, através destes, no desempenho da organização (VISSER, 2010).

Além disso, há evidências de que a influência das práticas de GRH no desempenho organizacional pode ser fortalecida quando as práticas são adequadas às exigências competitivas da empresa (YOUNDT et al., 1996). Observa-se, portanto, que um dos principais desafios enfrentados pelas organizações é combinar as estratégias voltadas para a qualidade com custos competitivos, atender as necessidades dos consumidores que estão sempre em mudança e ter capacidade de inovação (ALBUQUERQUE, 1992).

Dentre as várias opções estratégicas, Schuler e Jackson (1987) apresentaram uma tipologia de práticas de recursos humanos alinhadas às dimensões competitivas da empresa: inovação (produzir produtos e serviços diferentes dos concorrentes), qualidade (visa melhorar a qualidade do processo, dos produtos e serviços) e custo (busca a diferenciação pela apresentação de menor custo operacional). Sendo assim, as contribuições da GRH no desempenho organizacional podem ser observadas de algumas maneiras, especialmente nos resultados operacionais relacionados às variáveis custos, qualidade, flexibilidade, prazos, novos produtos e time-to-market (Quadro 2).

\subsection{Framework e hipóteses da pesquisa}

Na Figura 1 é apresentado of framework da pesquisa com os relacionamentos entre os construtos de gestão de recursos humanos e de desempenho operacional, que, segundo Schuler e Jackson (1987), devem estar alinhados. Com base nos conceitos apresentados na seção 2 originam-se as três hipóteses desta pesquisa, orientadas para o setor de autopeças e componentes automotivos:

- $\mathrm{H}_{1}$ - A gestão de recursos humanos pode ser observada pelas práticas de recrutamento e seleção, treinamento, avaliação de desempenho, recompensas e benefícios;
- $\mathrm{H}_{2}$ - A gestão de recursos humanos se relaciona positivamente com o desempenho operacional das organizações;

- $\mathrm{H}_{3}$ - O desempenho operacional no segmento de autopeças brasileiro pode ser observado pelas medidas de desempenho em redução de custos, time-to-market, lançamento de novos produtos, qualidade dos produtos, flexibilidade e prazos.

Os testes do framework e das hipóteses de pesquisa seguiram os procedimentos metodológicos descritos a seguir.

\section{Procedimentos metodológicos}

\subsection{Abordagem metodológica}

Esta pesquisa é quantitativa, baseada na estratégia survey, com a finalidade de teste de hipóteses, para a confirmação ou refutação do arcabouço teórico previamente revisado.

\subsection{Setor-alvo}

O segmento de autopeças e componentes automotivos integra o setor automotivo brasileiro, que teve seu início nos anos 1950. A evolução do setor apresenta os seguintes dados no Brasil: são 26 montadoras com 53 fábricas, abastecidas por centenas de empresas de autopeças, com capacidade produtiva instalada de 4,3 milhões de veículos e 109 mil máquinas agrícolas por ano, o que torna o Brasil um dos seis maiores produtores de veículos do mundo (ANFAVEA, 2011).

Atualmente, o setor emprega aproximadamente 1,5 milhão de pessoas, fatura anualmente mais de US\$ 107,6 bilhões (incluindo autopeças), tem uma participação direta no produto interno bruto (PIB) brasileiro de $5,2 \%$, mas que pode chegar a $22,5 \%$ do PIB se considerados os efeitos indiretos (ANFAVEA, 2011).

A indústria de autopeças, em conjunto com as montadoras, foi responsável por algumas importantes inovações, incluindo os motores bicombustíveis (Guia do Automóvel Brasil - ANFAVEA, 2011). Nesse sentido, o setor-alvo desta pesquisa é o segmento de autopeças e componentes automotivos, excluindo-se as empresas montadoras de automóveis e incluindo-se fabricantes de peças e as empresas "sistemistas", isto é, integradoras de peças e componentes, setor que, em 2010, faturou aproximadamente US $\$ 49,8$ bilhões e empregou cerca de 220 mil pessoas (SINDICATO..., 2011).

\subsection{Instrumento de coleta de dados}

Para a coleta de dados, foi planejado um instrumento do tipo questionário estruturado sobre os conceitos previamente revisados nas seções 2.1 e 2.2, elaborado 
segundo as recomendações contidas em Synodinos (2003).

O questionário possui, além de informações sobre a caracterização das empresas respondentes, dois blocos de assertivas, um para o construto gestão de recursos humanos e outro para desempenho operacional. No total, o questionário (Apêndice 1) tem cinco assertivas sobre gestão de recursos humanos (uma para cada prática de recursos humanos) e seis assertivas sobre desempenho operacional (uma para cada medida de desempenho operacional). A primeira versão do questionário foi submetida à validação de conteúdo, por meio da análise de cinco pesquisadores na área, além de adequação aos pressupostos conceituais, procedimento recomendado por Murillo-Luna, Garcés-Ayerbe e Rivera-Torres (2011). O questionário, em sua versão final, foi hospedado em um ambiente virtual especialmente elaborado para esta pesquisa. Embora a gestão de recursos humanos possa envolver temas como cultura organizacional e retenção de talentos etc., e embora o conceito de desempenho operacional possa incorporar mais variáveis sobre os temas de inovação e gestão da qualidade, optou-se por não avançar nessas possibilidades. Essa escolha se justifica, pois quanto mais variáveis se tem em um modelo conceitual, maior deverá ser a amostra coletada (HAIR JUNIOR. et al., 2005), conforme explicado na seção 3.4 abaixo.

Adotou-se uma escala Likert de 5 pontos, em que 1 representa discordo totalmente e 5 concordo totalmente, conforme o Apêndice 1 deste artigo.

\subsection{Histórico da coleta de dados}

Os dados desta pesquisa foram coletados entre os meses de outubro de 2010 e março de 2011. Primeiramente, foram coletados endereços de $e$-mail e informações telefônicas junto ao Sindicato Nacional da Indústria de Componentes para Veículos Automotores - SINDIPEÇAS, de 654 empresas do

Quadro 2. Variáveis relativas a medidas de desempenho operacional.

\begin{tabular}{|c|c|c|}
\hline Variáveis & Medidas & Fonte \\
\hline Custo & $\begin{array}{l}\text { Busca preço menor do que o dos } \\
\text { concorrentes, ou obtenção do menor } \\
\text { custo total de produção, ou aumento da } \\
\text { capacidade de produção. }\end{array}$ & $\begin{array}{l}\text { Hayes e Wheelwright (1984); Avella, Fernández } \\
\text { e Vázquez (2001); Christiansen et al. (2003); } \\
\text { Kaya, Caliskan e Gozlu (2007); Dal Pont, Furlan } \\
\text { e Vinelli (2008); Jabbour (2009); Chi, Kilduff e } \\
\text { Gargeya (2009); Furlan, Dal Pont e Vinelli (2011); } \\
\text { Gonzaléz-Benito (2005). }\end{array}$ \\
\hline Qualidade & $\begin{array}{l}\text { Fabricação de produção sem defeitos, ou } \\
\text { produtos duráveis. }\end{array}$ & $\begin{array}{l}\text { Hayes e Wheelwright (1984); Avella, Fernández } \\
\text { e Vázquez (2001); Christiansen et al. (2003); } \\
\text { Kaya, Caliskan e Gozlu (2007); Dal Pont, Furlan } \\
\text { e Vinelli (2008); Jabbour (2009); Chi, Kilduff e } \\
\text { Gargeya (2009); Furlan, Dal Pont e Vinelli (2011); } \\
\text { Gonzaléz-Benito (2005). }\end{array}$ \\
\hline Flexibilidade & $\begin{array}{l}\text { Mudanças rápidas de volumes de } \\
\text { produção, ou ampla variedade de } \\
\text { produtos, ou mudança rápida de mix de } \\
\text { produtos. }\end{array}$ & $\begin{array}{l}\text { Hayes e Wheelwright (1984); Avella, Fernández } \\
\text { e Vázquez (2001); Christiansen et al. (2003); } \\
\text { Kaya, Caliskan e Gozlu (2007); Dal Pont, Furlan } \\
\text { e Vinelli (2008); Jabbour (2009); Chi, Kilduff e } \\
\text { Gargeya (2009); Furlan, Dal Pont e Vinelli (2011); } \\
\text { Gonzaléz-Benito (2005). }\end{array}$ \\
\hline Novos produtos & $\begin{array}{l}\text { São produtos entrantes num determinado } \\
\text { mercado, com a finalidade de atrair } \\
\text { novos consumidores e/ou reter os atuais. } \\
\text { Relaciona-se a produtos com novas } \\
\text { características e funcionalidades. }\end{array}$ & $\begin{array}{l}\text { Hayes e Wheelwright (1984); Avella, Fernández } \\
\text { e Vázquez (2001); Christiansen et al. (2003); } \\
\text { Kaya, Caliskan e Gozlu (2007); Dal Pont, Furlan } \\
\text { e Vinelli (2008); Jabbour (2009); Chi, Kilduff e } \\
\text { Gargeya (2009); Furlan, Dal Pont e Vinelli (2011); } \\
\text { Gonzaléz-Benito (2005). }\end{array}$ \\
\hline Prazo & $\begin{array}{l}\text { Consiste num processo em que a empresa } \\
\text { busca atender de maneira eficiente os } \\
\text { prazos prometidos no atendimento dos } \\
\text { clientes. }\end{array}$ & $\begin{array}{l}\text { Hayes e Wheelwright (1984); Avella, Fernández, } \\
\text { Vázquez (2001); Christiansen et al. (2003); Kaya, } \\
\text { Caliskan e Gozlu (2007); Dal Pont, Furlan e } \\
\text { Vinelli (2008); Jabbour (2009); Chi, Kilduff e } \\
\text { Gargeya (2009); Furlan, Dal Pont e Vinelli (2011); } \\
\text { Gonzaléz-Benito (2005). }\end{array}$ \\
\hline Time-to-market & $\begin{array}{l}\text { Refere-se ao tempo de colocação de um } \\
\text { produto em um mercado, ou seja, desde } \\
\text { a concepção até a disponibilização no } \\
\text { ponto final de venda. }\end{array}$ & $\begin{array}{l}\text { Gonzalez-Benito (2005); González-Benito e } \\
\text { González-Benito (2005). }\end{array}$ \\
\hline
\end{tabular}




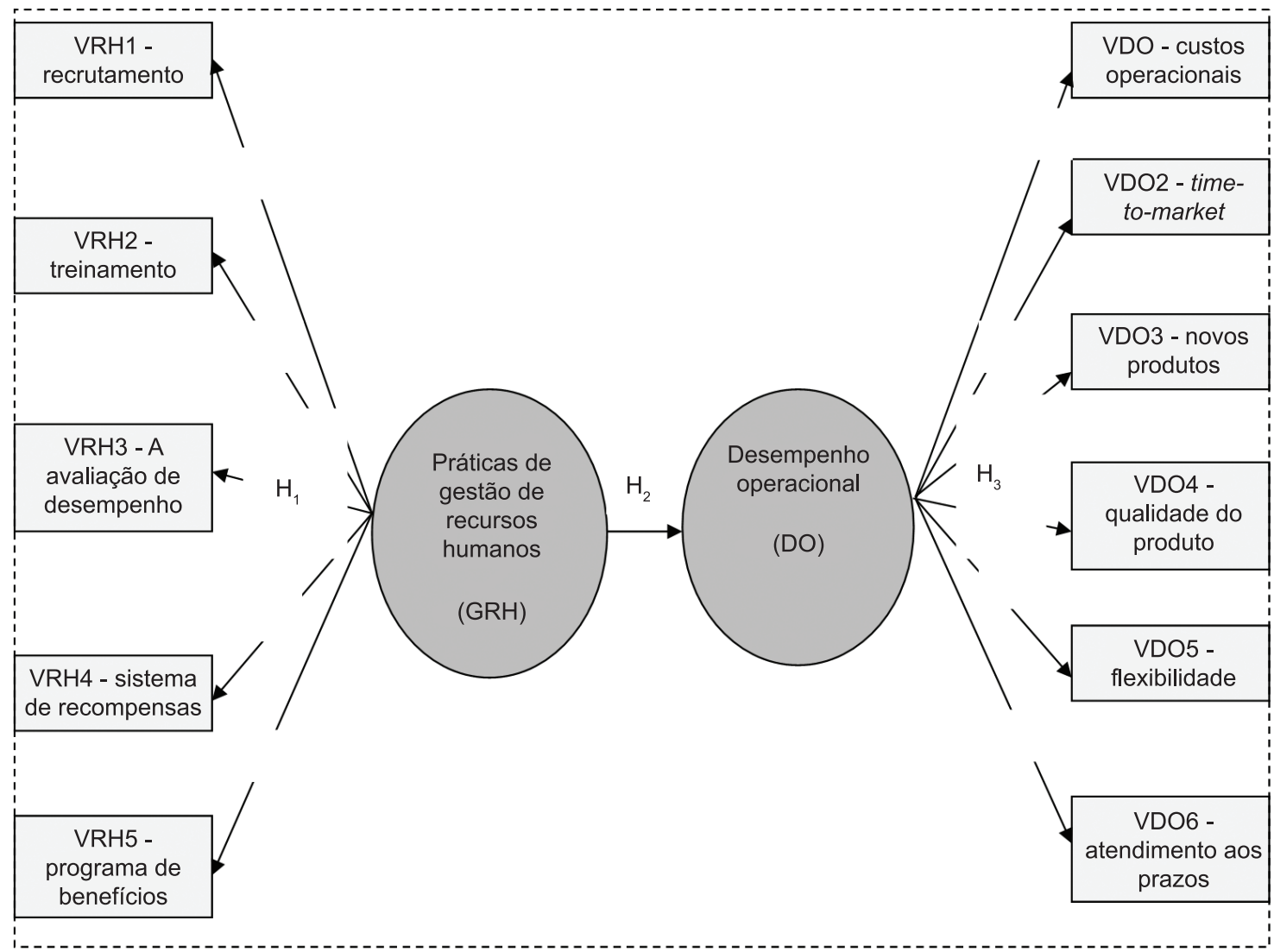

Figura 1. Framework relacional das hipóteses da pesquisa.

segmento de autopeças e componentes automotivos localizadas no Brasil. Foram enviados e-mails para essas empresas contendo uma breve explicação sobre a pesquisa e um convite para participação direcionado ao gestor de produção. No e-mail havia um link para direcionar o respondente-alvo diretamente para o questionário hospedado no ambiente virtual da pesquisa. Também foram realizadas ligações telefônicas direcionadas para o aumento do percentual de retorno de questionários válidos, em que se buscou contatar o funcionário responsável pela área de produção da empresa.

Assim, 72 questionários foram coletados por meio do site da pesquisa e 4 questionários coletados por meios alternativos, conforme solicitado pelos respectivos respondentes. Dos 76 questionários obtidos, um foi desconsiderado por estar incompleto. Totalizou-se, portanto, uma taxa de retorno de $11,11 \%$, (75 questionários válidos), valor considerado adequado frente aos percentuais indicados por Synodinos (2003) para pesquisas na área de produção e operações.

Como o modelo conceitual proposto possui 11 variáveis, segundo Hair Junior et al. (2005), deveriam ser coletados, no mínimo, 55 questionários. Outra recomendação é de que a amostra seja no mínimo 10 vezes superior à quantidade de variáveis contida no construto que tenha a maior quantidade de variáveis do modelo conceitual (ISMAIL; HAMID; IDRIS, 2012), isto é, um mínimo de 60 questionários para esta pesquisa. Percebe-se, portanto, que a quantidade de questionários coletada é adequada. Cada questionário preenchido alimentou automaticamente uma planilha de dados para posterior processamento estatístico.

Sobre o perfil da amostra, segundo a proposta de classificação de empresas por quantidade de funcionários recomendada pelo Sebrae, dos 75 questionários obtidos, $18,66 \%$ foram de pequenas empresas, $54,67 \%$, de médias empresas e 26,67\%, de grandes empresas. Esse perfil dá indícios de predominância de empresas de porte mais significativo na composição da amostra, podendo indicar também que as empresas maiores podem estar mais bem estruturadas para o engajamento em pesquisas acadêmicas.

\subsection{Análise dos resultados}

O framework de pesquisa (apresentado na seção 2.3) guiou o processo de análise de dados, que envolveu o uso de procedimentos estatísticos com o suporte de planilhas de dados do Software Statistical Package for Social Sciences da IBM (versão 19.0) e do Software Smart PLS 2.0. A seção 4 apresenta, detalhadamente, 
os procedimentos estatísticos associados a cada um dos resultados obtidos. Na seção 5, Discussões, é apresentado um quadro de consolidação da validade ou rejeição das hipóteses desta pesquisa.

\section{Resultados}

A redução dos dados de todas as variáveis, tanto do construto práticas de gestão de recursos humanos (GRH), quanto para o construto desempenho operacional (DO) foi realizada usando a análise de componentes principais, pelo método Varimax. Esse procedimento foi realizado independentemente para os construtos GRH e DO. Nesse primeiro procedimento, os principais indicadores de qualidade estatística foram:

- Adequação da amostra para cada fator individual pelo teste KMO (Kaiser-Meyer-Olkin);

- Cálculo do Alfa de Cronbach para cada fator;

- Teste Bartlett de esfericidade;

- Análise da diagonal principal da matriz anti-imagem;

- Comunalidades das variáveis;

- Eigenvalue para cada fator, em que foram extraídos os fatores com valores iguais ou superiores a 1,0

- A variância acumulada explicada.

A fim de refinar os resultados, a análise de componentes principais passou a revelar apenas cargas de variáveis acima de 0,6 , fatores com Eingenvalues maiores que 1 e coeficientes da diagonal da matriz anti-imagem maior do que 0,6. Verificou-se se a comunalidade das variáveis é igual ou superior a 0,5 para cada variável (HAIR JUNIOR et al., 2005). Em relação ao construto práticas de gestão de recursos humanos (GRH), apenas um fator foi formado, explicando uma variância acumulada aproximada de 68,12\%, com Eigenvalue de 2,72 e valores adequados na diagonal principal da matriz anti-imagem $(0,71 ; 0,61 ; 0,68 ; 0,63)$. O teste KMO, que verifica a adequação da amostra, foi de 0,662 , considerado adequado, assim como o valor obtido com o teste Bartlett de esfericidade $(141,41)$ e pelo Alfa de Conbrach $(0,84)$. O construto práticas de gestão de recursos humanos $(\mathrm{GRH})$ passou a ser formado pelas variáveis VRH1, VRH2, VRH3 e VRH4 (Tabela 1). A variável VRH5 foi excluída por apresentar baixa comunalidade $(0,38)$.

Após o refinamento do construto práticas de gestão de recursos humanos (GRH) acima relatado, percebe-se que a variável VRH2 - treinamento - foi aquela que obteve maior média entre as práticas de recursos humanos (Tabela 2).

Realizou-se também o teste de coeficientes de correlação de Pearson (Tabela 3), em que se verificou que todas as variáveis VRH1-VRH4 apresentam correlações significativas, com destaque para a relação entre VRH1 (recrutamento e seleção) e VRH2 (treinamento).

Tabela 1. Resultado da análise de componentes principais para GRH.

\begin{tabular}{ccc}
\hline Variáveis & Carga & Comunalidades \\
\hline VRH1 & 0,85 & 0,72 \\
VRH2 & 0,86 & 0,74 \\
VRH3 & 0,87 & 0,76 \\
VRH4 & 0,70 & 0,50 \\
\hline
\end{tabular}

Tabela 2. Média e desvio padrão das variáveis do construto GRH.

\begin{tabular}{ccc}
\hline Variáveis & Média & Desvio padrão \\
\hline VRH1 & 3,48 & 1,08 \\
VRH2 & 3,52 & 1,10 \\
VRH3 & 3,20 & 1,30 \\
VRH4 & 2,34 & 1,12 \\
\hline
\end{tabular}

Tabela 3. Correlação de Pearson para as variáveis do construto GRH.

\begin{tabular}{ccccc}
\hline & VRH1 & VRH2 & VRH3 & VRH4 \\
\hline VRH1 & 1 & & & \\
VRH2 & $0,71 *$ & 1 & & \\
VRH3 & $0,58^{*}$ & $0,72^{*}$ & 1 & \\
VRH4 & $0,49 *$ & $0,35^{*}$ & $0,55^{*}$ & 1 \\
\hline
\end{tabular}

$*$ p value $<0,05$.

Tabela 4. Resultado da análise de componentes principais para DO.

\begin{tabular}{ccc}
\hline Variáveis & Carga & Comunalidades \\
\hline VDO1 & 0,79 & 0,64 \\
VDO2 & 0,78 & 0,62 \\
VDO5 & 0,84 & 0,71 \\
VDO6 & 0,84 & 0,70 \\
\hline
\end{tabular}

Tabela 5. Média e desvio padrão das variáveis do construto DO.

\begin{tabular}{ccc}
\hline Variáveis & Média & Desvio padrão \\
\hline VDO1 & 4,25 & 0,89 \\
VDO2 & 4,11 & 0,89 \\
VDO5 & 4,34 & 0,74 \\
VDO6 & 4,36 & 0,78 \\
\hline
\end{tabular}

Tabela 6. Correlação de Pearson para as variáveis do construto DO.

\begin{tabular}{ccccc}
\hline & DO1 & DO2 & DO5 & DO6 \\
\hline VDO1 & 1 & & & \\
VDO2 & $0,58^{*}$ & 1 & & \\
VDO5 & $0,51^{*}$ & $0,52^{*}$ & 1 & \\
VDO6 & $0,53^{*}$ & $0,50^{*}$ & $0,70^{*}$ & 1 \\
\hline
\end{tabular}

$*$ p value $<0,05$. 
Tabela 7. Valores de confiabilidade e de validade do modelo estrutural.

\begin{tabular}{cccccc}
\hline Construtos & $\begin{array}{c}\text { Variância média } \\
\text { extraída (AVE) }\end{array}$ & $\begin{array}{c}\text { Confiabilidade } \\
\text { composta }\end{array}$ & $\begin{array}{c}\text { R } \\
\text { Square }\end{array}$ & Alfa de Cronbach & Comunalidade \\
\hline DO & 0,66 & 0,88 & 0,28 & 0,83 & 0,66 \\
GRH & 0,68 & 0,89 & - & 0,84 & 0,68 \\
\hline
\end{tabular}

Em relação ao construto desempenho operacional (DO), também apenas um fator foi formado, explicando uma variância acumulada aproximada de $66,79 \% \mathrm{com}$ Eigenvalue de 2,672 e valores adequados na diagonal principal da matriz anti-imagem (respectivamente $0,80 ; 0,80 ; 0,73 ; 0,73)$. O teste $\mathrm{KMO}$, que verifica a adequação da amostra, foi de 0,766 e é considerado adequado, assim como o valor obtido com o teste Bartlett de esfericidade $(116,10)$ e pelo Alfa de Conbrach $(0,83)$. O construto desempenho operacional (DO) passou a ser formado pelas variáveis VDO1, VDO2, VDO5 e VDO6. As variáveis VDO3 e VDO4 foram excluídas da análise por apresentarem comunalidades, respectivamente, de 0,38 e 0,43 (Tabela 4).

Após o refinamento do construto desempenho operacional (DO) acima relatado, observa-se que a variável VDO6 (capacidade de atendimento aos prazos estabelecidos pelos clientes) foi aquela que obteve maior média entre as medidas de desempenho operacional (Tabela 5).

Realizou-se também o teste de coeficientes de correlação de Pearson (Tabela 6), onde se verifica que todas as variáveis VDO1, VDO2, VDO5 e VDO6 apresentam correlações significativas, com destaque para a relação entre VDO5 (flexibilidade para se adaptar aos clientes) e VDO6 (capacidade para atender aos prazos dos clientes).

Em seguida, Structural Equation Modeling - Partial Least Squares (SEM-PLS) foi utilizada. A modelagem de equações estruturais por meio de PLS é considerada uma análise multivariada de segunda geração. Ela é especialmente útil quando se trabalha com teoria complexa (relacionando conceitos tradicionais, como recursos humanos e desempenho operacional) ou e em estágios iniciais de desenvolvimento. Frente às demais técnicas estatísticas disponíveis, a modelagem de equações estruturais é vantajosa, pois: a) permite aos pesquisadores o teste de modelos conceituais mais complexos, garantindo uma análise estatística do modelo de forma mais robusta e holística (ISMAIL; HAMID; IDRIS, 2012); b) permite a análise da relação entre um amplo grupo de variáveis simultaneamente (HAIR JUNIOR; RINGLE; SARSTED, 2011). Para aplicá-la nesta pesquisa, um modelo estrutural (Figura 2) foi criado contendo os construtos obtidos a partir da análise de componentes principais, como explicado anteriormente. O objetivo desse procedimento foi testar a validade e confiabilidade
Tabela 8. Cargas cruzadas para avaliação da validade discriminante.

\begin{tabular}{lll}
\hline & DO & RH \\
\hline VDO1 & 0,78 & 0,40 \\
VDO2 & 0,76 & 0,34 \\
VDO5 & 0,87 & 0,55 \\
VDO6 & 0,83 & 0,39 \\
VRH1 & 0,44 & 0,85 \\
VRH2 & 0,49 & 0,86 \\
VRH3 & 0,39 & 0,85 \\
VRH4 & 0,41 & 0,71 \\
\hline
\end{tabular}

do modelo. As análises foram realizadas usando o software SmartPLS 2.03 (SOSIK et al., 2009).

Indicadores de boa qualidade para o modelo proposto foram alcançados em termos de variância média extraída (validade convergente), confiabilidade composta, Alfa de Cronbach e comunalidades, tanto para o construto GRH, quanto para o DO. Para checar se a confiabilidade (que apura a precisão com que o construto mede exatamente aquilo que realmente deveria medir) e a validade (que testa a relação de uma variável com outra variável de um mesmo construto) são satisfatórias, o valor de confiabilidade composta deve ser superior a 0,7 , enquanto o valor da validade convergente deve ser superior a 0,5. A confiabilidade de construto foi avaliada utilizando confiabilidade composta. Já validade convergente foi analisada pela variância média extraída. A Tabela 7 mostra que todos os valores de confiabilidade composta são superiores a 0,7 e todos os valores de variância média extraída são superiores a 0,5 (FOLTZ, 2008). Os coeficientes de Alfa de Cronbach e as comunalidades também são considerados adequados.

Uma das formas para garantir a validade discriminante é verificar se as variáveis realmente possuem cargas mais elevadas em seus fatores de origem. Essa análise obteve resultados adequados Tabela 8.

Com vistas ao teste de robustez do modelo, um bootstrap de 1.000 subamostras foi utilizado para estimar a significância estatística das relações entre as variáveis propostas e os construtos (Figura 3).

Todas as relações do modelo são estatisticamente válidas ao nível de significância, $p$ value menor ou igual a 0,01 , conforme a Tabela 9 . 


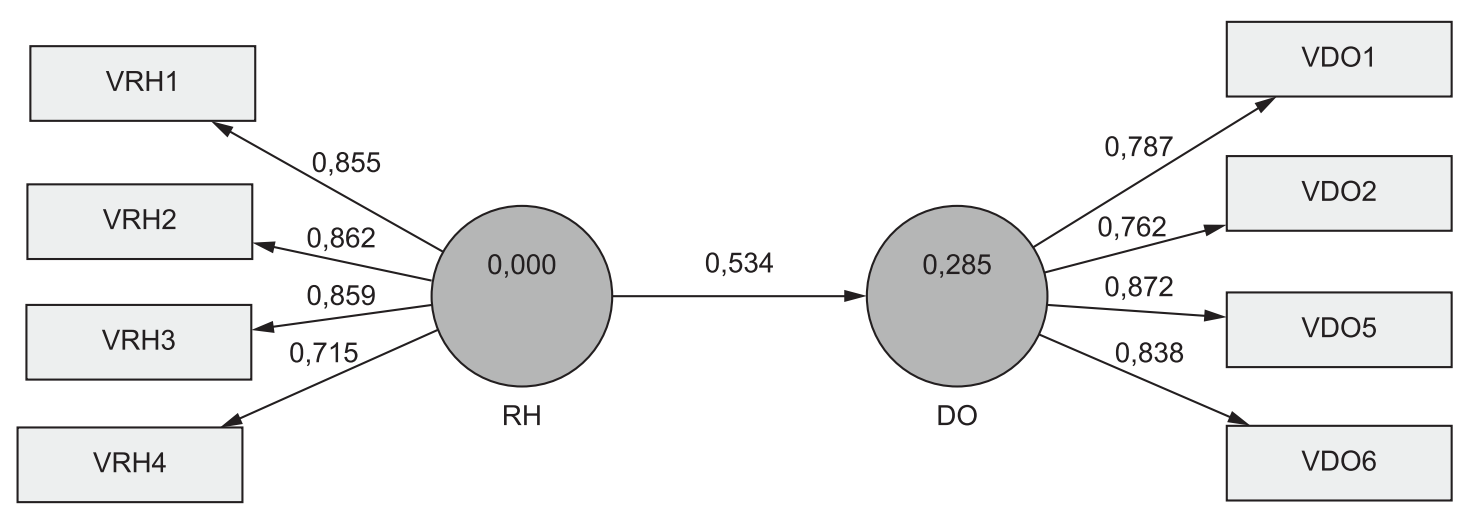

Figura 2. Modelo estrutural.

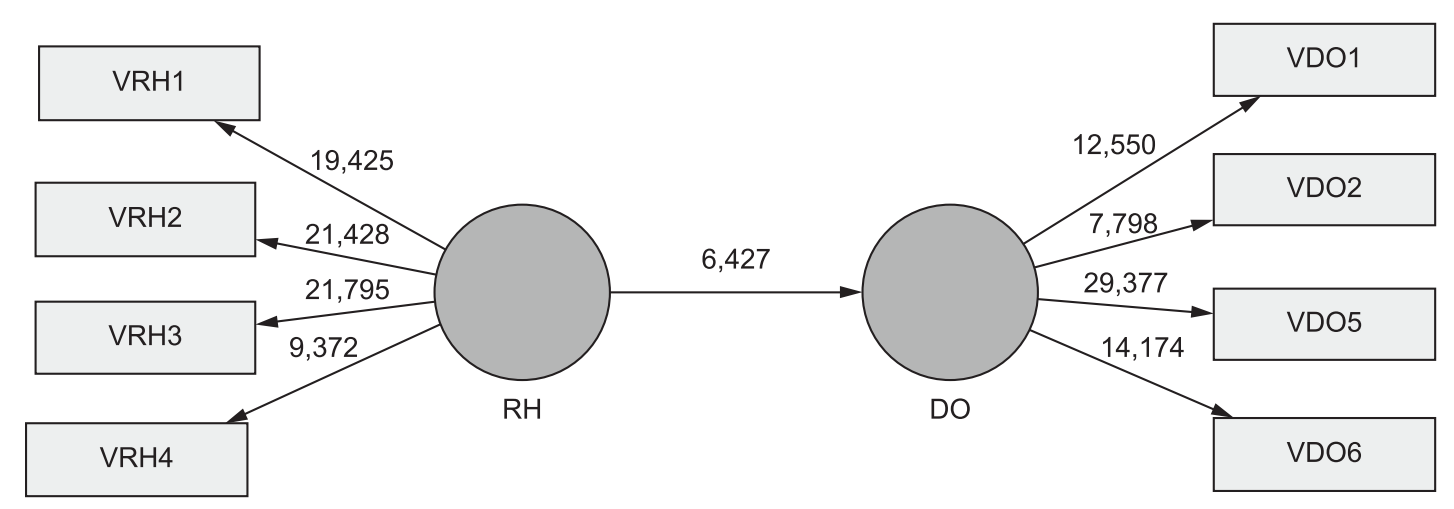

Figura 3. Modelo estrutural com bootstrapping de 1.000 subamostragens.

Tabela 9. Significância dos coeficientes dos relacionamentos do modelo.

\begin{tabular}{cccc}
\hline Relacionamentos & Coeficiente & Teste t & Significância \\
\hline $\mathrm{RH} \rightarrow \mathrm{DO}$ & 0,53 & 6,42 & $*$ \\
$\mathrm{VRH} 1 \leftarrow \mathrm{RH}$ & 0,85 & 19,42 & $*$ \\
$\mathrm{VRH} 2 \leftarrow \mathrm{RH}$ & 0,86 & 21,42 & $*$ \\
$\mathrm{VRH} 3 \leftarrow \mathrm{RH}$ & 0,85 & 21,79 & $*$ \\
$\mathrm{VRH} 4 \leftarrow \mathrm{RH}$ & 0,71 & 9,371 & $*$ \\
$\mathrm{VDO} 1 \leftarrow \mathrm{DO}$ & 0,78 & 7,54 & $*$ \\
$\mathrm{VDO} 2 \leftarrow \mathrm{DO}$ & 0,76 & 29,37 & $*$ \\
$\mathrm{VDO} 5 \leftarrow \mathrm{DO}$ & 0,87 & 14,17 & $*$ \\
\hline
\end{tabular}

$*$ minimamente $\mathrm{p}$ value $\leq 0,01$.

\section{Discussão}

O Quadro 3 consolida a validade das hipóteses de pesquisa declaradas na seção 2.3 deste artigo. Verifica-se que a $\mathrm{H} 1$ foi apenas parcialmente validada, uma vez que a VRH5 não se mostrou estatisticamente adequada. Não porque o setor sob análise não possua - nem adote - práticas adequadas de benefícios, mas sim porque tal variável não foi significativa o suficiente para ser estatisticamente adequada. Por outro lado, a maior parte das práticas de gestão de recursos humanos que constam na revisão da literatura foi verificada na prática, com destaque para a VRH2 (prática de treinamento), que torna o trabalhador mais apto ao desempenho das atividades laborais cotidianas de um estabelecimento industrial, como sugere Borges-Andrade (2002). As demais práticas de recursos humanos, como recrutamento e seleção, avaliação de desempenho e recompensas também se mostraram estatisticamente adequadas.

$\mathrm{O}$ resultado mais relevante foi a verificação de influência positiva do construto práticas de gestão de recursos humanos no desempenho operacional das 
Quadro 3. Hipóteses, nível de validação e justificativas.

\begin{tabular}{|c|l|c|c|}
\hline Hipótese & \multicolumn{1}{|c|}{ Assertiva } & Nível de validade & Justificativa \\
\hline $\mathrm{H}_{1}$ & $\begin{array}{l}\text { A gestão de recursos humanos no segmento de } \\
\text { autopeças brasileiro pode ser observada pelas práticas } \\
\text { de recrutamento e seleção, treinamento, avaliação de } \\
\text { desempenho, recompensas e benefícios }\end{array}$ & $\begin{array}{c}\text { Apenas } \\
\text { parcialmente } \\
\text { válida }\end{array}$ & $\begin{array}{c}\text { VRH5 estatisticamente } \\
\text { não adequada }\end{array}$ \\
\hline $\mathrm{H}_{2}$ & $\begin{array}{l}\text { A gestão de recursos humanos influencia positivamente o } \\
\text { desempenho operacional }\end{array}$ & Válida & $\begin{array}{c}\text { Nível de significância } \\
\text { adequado }\end{array}$ \\
\hline $\mathrm{H}_{3}$ & $\begin{array}{l}\text { O desempenho operacional no setor brasileiro de autopeças } \\
\text { e componentes automotivos pode ser observado pelas } \\
\text { medidas de desempenho em redução de custos, time-to- } \\
\text { market, lançamento de novos produtos, qualidade dos } \\
\text { produtos, flexibilidade e atendimento aos prazos }\end{array}$ & $\begin{array}{c}\text { Apenas } \\
\text { parcialmente } \\
\text { válida }\end{array}$ & $\begin{array}{c}\text { VDO3 e VDO4 } \\
\text { estatisticamente não } \\
\text { adequadas }\end{array}$ \\
\hline
\end{tabular}

empresas analisadas. Em outras palavras, a validação da hipótese 2 confirma os argumentos dos estudiosos da área (SCHULER; JACKSON, 1987; BECKER; GERHART, 1996; ULRICH, 1997; BECKER; HUSELID, 2006; JANSSENS; STEYAER, 2009) de que a gestão de recursos humanos tende a influenciar o desempenho das organizações. Adiciona-se, portanto, ao estado-da-arte do tema uma perspectiva brasileira, de um importante setor industrial nacional.

Percebe-se, também, que a hipótese 3 , sobre a configuração do desempenho operacional, foi apenas parcialmente validada, uma vez que as variáveis VDO3 e VDO4 não apresentaram valores estatísticos significativos adequados à sua manutenção no modelo testado. Esse resultado não indica, entretanto, que gestão da qualidade (qualidade do produto) e da inovação (novos produtos) não sejam relevantes para as empresas da amostra. Revelam apenas que essas variáveis não alcançaram significância estatística. Embora novas pesquisas sejam necessárias para aprofundar entendimento sobre o comportamento dessas variáveis, pode-se sugerir que os produtos do setor de autopeças tendem a seguir as especificações de lançamento e qualidade estabelecidas pelas montadoras; e que a qualidade vem se tornando um fator qualificador e não ganhador de contratos de fornecimento, o que poderia explicar o comportamento estatístico dessas variáveis.

\section{Conclusões}

A proposta desta pesquisa foi verificar se a gestão de recursos humanos influencia positivamente o desempenho das operações de empresas do segmento de autopeças e componentes automotivos. Para tanto, elaborou-se um framework com hipóteses de pesquisa que foram testadas à luz da realidade de 75 empresas do setor no Brasil. Após o processamento e análise dos resultados por meio de modelagem de equações estruturais, os principais resultados foram:

- De fato, a gestão de recursos humanos influencia positivamente o desempenho operacional do setor;
- Tanto a gestão de recursos humanos quanto o desempenho operacional adotam algumas práticas/medidas indicadas pela literatura especializada que não foram estatisticamente consideradas válidas e que merecem ser analisadas em profundidade por pesquisas futuras.

Tendo em vista as características desta pesquisa e dos resultados obtidos, sugere-se que pesquisas futuras avancem sobre os seguintes temas:

- Pesquisar mais profundamente a gestão de benefícios nas empresas do setor mencionado;

- Verificar outras dimensões que compõem os conceitos de inovação e de gestão da qualidade no âmbito do desempenho operacional das empresas do setor;

- Analisar a validade da hipótese de relacionamento entre recursos humanos e desempenho operacional não somente para empresas de autopeças e componentes automotivos, mas também para empresas montadoras de automóveis.

Por fim, cabe registrar que esta pesquisa possui limitações. Por isso, os resultados devem ser considerados ponderando-se que:

- Diversas práticas de gestão de recursos humanos (por exemplo, desenvolvimento de cultura e clima organizacional; e gestão de carreira, sucessão e retenção de talentos; e desenvolvimento dos processos e da estrutura organizacional) foram excluídas da pesquisa, para se garantir um questionário mais parcimonioso e adequado à expectativa em relação ao tamanho da amostra;

- Outras dimensões do desempenho operacional (por exemplo, inovação em processos, serviços e organizacionais; e qualidade do projeto do produto e da execução operacional) não foram incluídas no questionário porque gerariam uma 
quantidade de assertivas muito maior do que a compatível com a amostra da pesquisa;

- A amostra sob análise considerou apenas empresas do setor de autopeças e componentes/ sistemas automotivos. Esse segmento tende a ser composto por empresas de médio e grande porte intensivas em tecnologia.

\section{Referências}

ALBUQUERQUE, L. G. Competitividade e recursos humanos. Revista de Administração da USP, v. 27, n. 4, p. 16-29, 1992.

ASSOCIAÇÃO NACIONAL DOS FABRICANTES DE VEÍCULOS AUTOMOTORES - ANFAVEA. Disponível em: <http://www.anfavea.org.br>. Acesso em: jun. 2011.

ARTHUR, J. B. Effects of human resource systems on manufacturing performance and turnover. Academy of Management Journal, v. 37, n. 3, p. 670-87, 1994. http://dx.doi.org/10.2307/256705

AVELLA, L.; FERNÁNDEZ, E.; VÁZQUEZ, C. J. Analysis of manufacturing strategy as an exploratory factor of competitiveness in the large Spanish industrial firm. International Journal of Production Economics, v. 72, p. 139-157, 2001. http://dx.doi.org/10.1016/ S0925-5273(00)00099-2

BATEMAN, T. S.; SNELL, S. A. Administração: construindo vantagens competitivas. Tradução de Celso A. Rimoli. São Paulo: Atlas, 1998.

BECKER, B. E.; HUSELID, M. A. Strategic Human Resources Management: Where Do We Go From Here? Journal of Management, v. 32, n. 6, p. 898-925, 2006. http://dx.doi.org/10.1177/0149206306293668

BECKER, B.; GERHART, B. The impact of Human Resource Management on Organizational Performance: Progress and Prospects. The Academy of Management Journal, v. 39, n. 4, p. 779-801, 1996. http://dx.doi. org/10.2307/256712

BORGES-ANDRADE, J. E. Desenvolvimento de medidas em avaliação de treinamento. Estudos de Psicologia, v. 7, número especial, p. 31-43, 2002.

BOSELIE, P., DIETZ, G.; BOON, C. Commonalities and contradictions in HRM and performance research. Human Resource Management Journal, v. 15, n. 3, p. 67-94, 2005. http://dx.doi.org/10.1111/j.1748-8583.2005. tb00154.x

BOUDREAU, J. et al. On the interface between operations and Human Resource Management. Manufacturing \& Services Operations Management, v. 5, n. 3, p.179-202. 2003. http://dx.doi.org/10.1287/msom.5.3.179.16032

BREWSTER, C.; MORLEY, M.; BUCIUNIENE, I. The reality of human resource management in Central and Eastern Europe. Baltic Journal of Management, v. 5, n. 2, p. 145-155, 2010. http:// dx.doi.org/10.1108/17465261011045098

CABRERA, E. F.; CABRERA, A. Strategic Human Resource Evaluation. Human Resource Planning, v. 26, n. 1, p. 41-50, 2003.

CHI, T.; KILDUFF, P. P. D.; GARGEYA, V. B. Alignment between business environment characteristics, competitive priorities, supply chain structures, and firm business performance. International Journal of Productivity and Performance Management, v. 58, n. 7, p. 645-669, 2009. http://dx.doi.org/10.1108/17410400910989467

CHRISTIANSEN, T. et al. A mapping of competitive priorities, manufacturing practices, and operational performance in groups of Danish manufacturing companies. International Journal of Operations \& Production Management, v. 23, n. 10, p. 1163-1183, 2003. http://dx.doi.org/10.1108/01443570310496616

COSTA, A. C. F.; SOUZA, S. S.; SILVA, L. C. T. Investigação sobre a satisfação do usuário dos serviços prestados pelo metrô de são Paulo: um estudo exploratório, descritivo e ilustrativo com a utilização do modelo de equações estruturais. Revista de Gestão da USP, v. 15, número especial, p. 93-108, 2008.

DAFT, R. L. Administração. 4. ed. Tradução de Fernando Gastaldo Morales.LTC, 1999.

DAL PONT, G.; FURLAN, A.; VINELLI, A. Interrelationships among lean bundles and their effects on operational performance. Operations Management Research, v. 1, p. 150-158, 2008. http://dx.doi. org/10.1007/s12063-008-0010-2

DELEEY, J.; DOTY, D.H. Modes of theorizing in strategic human resource management: tests of universalistic, contingency and configurationally performance predictions. The Academy of Management Journal, v. 39, n. 4, p. 802-835, 1996. http://dx.doi. org/10.2307/256713

DESSLER, G. Administração de recursos humanos. 2. ed. Tradução de Cecília Leão Oderich. São Paulo: Pearson Prentice Hall, 2003.

DEWETTINCK, K.; REMUE, J. Contextualizing HRM in comparative research: The role of the Cranet network. Human Resource Management Review, v. 21, p. 37-49, 2011. http://dx.doi.org/10.1016/j.hrmr.2010.09.010

DUTRA, J. S. Competências: conceitos e instrumentos para a gestão de pessoas na empresa moderna. São Paulo: Atlas, 2004.

DUTRA, J. S. Gestão de pessoas: modelo, processos, tendências e perspectivas. São Paulo: Atlas, 2008.

DYER, L.; REEVES, T. Human resource strategies and firm performance: What do we know and where do we need to go? The International Journal of Human Resource Management, v. 6, n. 3, p. 656-670, 1995. http://dx.doi.org/10.1080/09585199500000041

FERGUSON, K. L.; REIO JUNIOR, T. G. Human resource management systems and firm performance. Journal of Management Development, v. 29, n. 5, p. 471-494, 2010. http://dx.doi.org/10.1108/02621711011039231

FISCHER, A. L. Um resgate conceitual e histórico dos modelos de gestão de pessoas. In: FLEURY, M. T. L. (Org.). As pessoas na organização. 9. ed. São Paulo: Gente, 2002. p. 11-33.

FLEURY, M. T. L. (Org.). As pessoas na organização. 9. ed. São Paulo: Gente, 2002.

FOLTZ, C. B. Why users (fail to) read computer usage policies. Industrial Management \& Data Systems, v. 108, n. 6, p. 701-12, 2008. http://dx.doi. org/10.1108/02635570810883969 
FURLAN, A.; DAL PONT, G.; VINELLI, A. On the complementarily between internal and external justin-time bundles to build and sustain high performance manufacturing. International Journal of Production Economics, v. 133, n. 2, p. 489-495, 2011. http://dx.doi. org/10.1016/j.ijpe.2010.07.043

GONZÁLEZ-BENITO, J. A study of the effect of manufacturing proactivity on business performance. International Journal of Operations \& Production Management, v. 25, n. 3, p. 222-241, 2005. http:// dx.doi.org/10.1108/01443570510581844

GONZÁLEZ-BENITO, J.; GONZÁLEZ-BENITO, O. Environmental proactivity and business performance: na empirical analysis. Omega: The International Journal of Management Science, v. 33, p. 1-15, 2005.

GOODERHAM, P.; RINGDAL, K.; PARRY, E. The impact of human resource management practices on the performance of European firms. In: COPENHAGEN BUSINESS SCHOOL CONFERENCE ON HRM AND KNOWLEDGE-RELATED PERFORMANCE, 2006, Copenhagen. Proceedings... Copenhagen, 2006.

GUEST, D. E. Human resource management and performance: still searching for some answers. Human Resource Management Journal, v. 21, n. 1, p. 3-13, 2011. http://dx.doi.org/10.1111/j.1748-8583.2010.00164.x

HAIR JUNIOR, J. F. et al. Fundamentos de métodos de pesquisa em administração. Porto Alegre: Bookman, 2005.

HAIR JUNIOR, J. F.; RINGLE, C. M.; SARSTEDT, M. PLS-SEM: indeed a silver bullet. Journal of Marketing Theory and Practice, v. 19, n. 2, p. 139-151, 2011. http://dx.doi.org/10.2753/MTP1069-6679190202

HALL, R. Organizations: structures, processes, and outcomes. Pearson: Upper Saddle. 2004.

HAYES, R. H.; WHEELWRIGHT, S. C. Restoring our competitive edge - competing through manufacturing. John Wiley \& Sons, 1984.

HIPOLITO, J. A. M. Sistema de recompensas: uma abordagem atual. In: FLEURY, M. T. L. (Org.). As pessoas na organização. 9. ed. São Paulo: Gente, 2002.

HUSELID, M. A. The impact $\mathrm{f}$ human resource management practices on turnover, productivity and corporate financial performance. Academy of Management Journal, v. 8, n. 3, p. 835-872, 1995.

HUSELID, M. A.; JACKSON, S. E.; SCHULER, R. S. Technical and strategic human resource management effectiveness as determinants of firm performance. Academy of Management Journal, v. 40, n. 1, p. 171-188, 1997. http://dx.doi.org/10.2307/257025

ISMAIL, I. R.; HAMID, R. A.; IDRIS, F. PLS application in Journals of Operations Management: a review. In: GLOBAL CONFERENCE ON OPERATIONS AND SUPPLY CHAIN MANAGEMENT, 2012, Bandung. Proceedings... Bandung, 2012. p. 1-6.

JABBOUR, A. B. L. S. Prioridades competitivas da produção e práticas de gestão da cadeia de suprimentos: uma survey no setor eletrônico brasileiro. 2009. Tese (Doutorado em Engenharia de Produção)Universidade Federal de São Carlos, São Carlos, 2009.

JACKSON, S. E.; SCHULER, R. S. Understanding human resource management in the context of organizations and their environments. Annual Review of Psychology, v. 46, p. 237-264, 1995. PMid:19245335. http://dx.doi. org/10.1146/annurev.ps.46.020195.001321

JAMROG, J.; OVERHOLT, M. H. Building a strategic HR function: continuing the evolution. Human Resource Planning, v. 27, n. 1, p. 51-63, 2004.

JANSSENS, M.; STEYAERT, C. HRM and Performance: A plea for reflexivity in HRM studies. Journal of Management Studies, v. 46, n. 1, p. 143-155, 2009. http://dx.doi.org/10.1111/j.1467-6486.2008.00812.x

KAYA, E.; CALISKAN, F. D.; GOZLU, S. Manufacturing performance criteria: an AHP application in a textile company. In: PORTLAND INTERNATIONAL CENTER FOR MANAGEMENT OF ENGINEERING AND TECHNOLOGY - PICMET, 2007, Portland. Proceedings... Portland, 2007.

KOUHY, R., VEDD, R.; YOSHIKAWA, T. Human resource policies, management accounting and organisational performance. Journal of Human Resource Costing \& Accounting, v. 13, n. 3, p. 245-263, 2009. http:// dx.doi.org/10.1108/14013380910995520

LACOMBE, B. M. B.; ALBUQUERQUE, L. G. Avaliação e Mensuração de resultados em gestão de pessoas: um estudo com as maiores empresas instaladas no Brasil. Revista de Administração, v. 43, n. 1, p. 5-16, 2008.

LENGNICK-HALL, M. L. et al. Strategic human resource management: The evolution of the field. Human Resource Management Review, v. 19, n. 2, p. 64-85, 2009. http://dx.doi.org/10.1016/j.hrmr.2009.01.002

LIMONGI-FRANÇA, A. C. Práticas de recursos humanos conceitos, ferramentas e procedimentos. São Paulo: Atlas, 2007.

MURILLO-LUNA, J. L.; GARCÉS-AYERBE, C.; RIVERA-TORRES, P. Barriers to the adoption of proactive environmental strategies. Journal of Cleaner Production, v. 19, p. 1417-1425, 2011. http://dx.doi. org/10.1016/j.jclepro.2011.05.005

MARRAS, J. P. Administração de Recursos Humanos: do operacional ao estratégico. São Paulo: Futura, 2001.

MILKOVICH, G. T.; BOUDREAU, J. W. Administração de recursos humanos. São Paulo: Atlas, 2006.

OLIVEIRA, P. W. S.; LEONE, R. G. J. Gestão estratégica de benefícios: proposição de um modelo matemático para assistência médica dos funcionários. RAM - Revista de Administração Mackenzie, v. 9, n. 2, p. 104-127, 2008.

PAAUWE. J. HRM and performance: achievements, methodological issues and prospects. Journal of Management Studies, v. 46, n. 1, 2009.

ROBBINS, S. P.; DECENZO, D. A. Fundamentos de administração: conceitos essenciais e aplicações. 4. ed. Tradução de Robert Brian Taylor. Revisão técnica de Reinaldo O. da Silva. São Paulo: Pearson Prentice Hall, 2004.

SCHULER, R.; JACKSON, S. Linking competitive strategies and human resource management pratices. Academy of Management Executive, v. 1, n. 3, p. 207-219, 1987. http://dx.doi.org/10.5465/AME.1987.4275740

SILVEIRA, A. C. et al. Gestão Estratégica de Pessoas São Paulo: FGV, 2010

SINDICATO NACIONAL DA INDÚSTRIA DE COMPONENTES PARA VEÍCULOS 
AUTOMOTORES - SINDIPEÇAS; ASSOCIAÇÃO BRASILEIRA DA INDÚSTRIA DE AUTOPEÇAS - ABIPEÇAS. Desempenho do setor de autopeças 2011. SINDIPEÇAS; ABIPEÇAS, 2011. Disponível em: <http://www.virapagina.com. br/sindipecas2011/>. Acesso em: 16 abr. 2012

SOSIK, J. J.; KAHAI, S. S.; PIOVOSO, M. J. Silver bullet or voodoo statistics?: A primer for using least squares data analytic technique in group and organization research. Group \& Organization Management, v. 35, n. 5, p. 5-36, 2009. http://dx.doi. org/10.1177/1059601108329198

SROGGINS, W. A; BENSON, P. G. International human resource management: diversity, issues and challenges. Personal Review, v. 39, n. 4, p. 409-413, 2010. http:// dx.doi.org/10.1108/00483481011045380

STONER, J. A. F.; FREEMAN, R. E. Administração. 5. ed. Tradução de Alice Alves Calado. LTC, 1999.

SYNODINOS, N. E. The "art" of questionnaire construction: some important considerations for manufacturing studies. Integrated Manufacturing Systems, v. 14, n. 3, p. 221-237, 2003. http://dx.doi. org/10.1108/09576060310463172

TÜRK, K.; ROOLAHT, T. Appraisal and compensation of the academic staff in Estonian public and private universities: a comparative analysis. Trames, v. 11, n. 2, p. 206-222, 2007.

ULRICH, D. Measuring human resources: an overview of practice and a prescription for results. Human Resource
Management, v. 36, n. 3, p. 303-320, 1997. http://dx.doi. org/10.1002/(SICI)1099-050X(199723)36:3<303::AIDHRM3>3.0.CO;2-\#

VERGARA, S. C. Gestão de pessoas. Editora Atlas: São Paulo, 2011.

VISSER, M. Configurations of human resource practices and battlefield performance: A comparison of two armies. Human Resource Management Review, v. 20, n. 4, p. 340-349, 2010. http://dx.doi.org/10.1016/j. hrmr.2010.04.002

VOORDE, K. V. D.; PAAUWE, P.; VELDHOVEN, M. V. Predicting business unit performance using employee surveys: monitoring HRM-related changes. Human Resource Management Journal, v. 20, n. 1, p. 44-63, 2010. http://dx.doi. org/10.1111/j.1748-8583.2009.00114.x

WOMACK, P. J.; JONES, D. T.; ROOS, D. A máquina que mudou o mundo. Rio de Janeiro: Elsevier, 2004. WRIGHT, P. M.; GARDNER, T. M.; MOYNIHAN, L. M. The impact of HR practices on the performance of business units. Human Resource Management Journal, v. 13, n. 3, p. 21-36, 2003. http://dx.doi. org/10.1111/j.1748-8583.2003.tb00096.x

YOUNDT, M. A. et al. Human resource management, manufacturing strategy and firm performance. Academy of Management Journal, v. 39, n. 4, 1996, p. 836-866. http://dx.doi.org/10.2307/256714 


\section{Apêndice 1}

Assinale a alternativa que melhor expressa o seu nível de concordância em relação a afirmações sobre o desempenho operacional da sua empresa (últimos 3 anos).

\begin{tabular}{|l|l|l|l|l|l|}
\cline { 2 - 5 } \multicolumn{1}{c|}{} & $\begin{array}{c}\text { Discordo } \\
\text { totalmente }\end{array}$ & $\begin{array}{c}\text { Discordo } \\
\text { (parcialmente) }\end{array}$ & $\begin{array}{c}\text { Nem discordo, } \\
\text { nem concordo }\end{array}$ & $\begin{array}{c}\text { Concordo } \\
\text { (parcialmente) }\end{array}$ & $\begin{array}{c}\text { Concordo } \\
\text { totalmente }\end{array}$ \\
\hline $\begin{array}{l}\text { Reduzimos os custos } \\
\text { operacionais (fornecimento, } \\
\text { produção e entrega) }\end{array}$ & & & & \\
\hline $\begin{array}{l}\text { Reduzimos o tempo necessário } \\
\text { para criar e entregar novos } \\
\text { produtos }\end{array}$ & & & & & \\
\hline $\begin{array}{l}\text { Aumentamos o lançamento de } \\
\text { novos produtos }\end{array}$ & & & & & \\
\hline $\begin{array}{l}\text { Aumentamos a qualidade do } \\
\text { produto (atendimento aos } \\
\text { requisitos) }\end{array}$ & & & & & \\
\hline $\begin{array}{l}\text { Aumentamos a flexibilidade } \\
\text { para nos adaptar aos diferentes } \\
\text { pedidos dos clientes }\end{array}$ & & & & & \\
\hline $\begin{array}{l}\text { Melhoramos nossa capacidade } \\
\text { para atender os prazos dos } \\
\text { clientes }\end{array}$ & & & & & \\
\hline
\end{tabular}

Assinale a alternativa que corresponde ao nível de qualidade das práticas de recursos humanos.

\begin{tabular}{|l|l|l|l|l|l|}
\cline { 2 - 6 } \multicolumn{1}{c|}{} & $\begin{array}{c}\text { Discordo } \\
\text { totalmente }\end{array}$ & $\begin{array}{c}\text { Discordo } \\
\text { (parcialmente) }\end{array}$ & $\begin{array}{c}\text { Nem discordo, } \\
\text { nem concordo }\end{array}$ & $\begin{array}{c}\text { Concordo } \\
\text { (parcialmente) }\end{array}$ & $\begin{array}{c}\text { Concordo } \\
\text { totalmente }\end{array}$ \\
\hline $\begin{array}{l}\text { O processo de recrutamento e } \\
\text { seleção é eficiente e eficaz }\end{array}$ & & & & & \\
\hline $\begin{array}{l}\text { O processo de treinamento é } \\
\text { eficiente e eficaz }\end{array}$ & & & & & \\
\hline $\begin{array}{l}\text { O sistema de avaliação de } \\
\text { desempenho do funcionário é } \\
\text { eficiente e eficaz }\end{array}$ & & & & & \\
\hline $\begin{array}{l}\text { O sistema de recompensas } \\
\text { (salário e comissão) é } \\
\text { eficiente e eficaz }\end{array}$ & & & & & \\
\hline $\begin{array}{l}\text { O sistema de benefícios } \\
\text { (saúde, lazer) é eficiente e } \\
\text { eficaz }\end{array}$ & & & & & \\
\hline
\end{tabular}

